\title{
"Is there an Oslo, France, mr. Ashbery?": las intervenciones públicas de John Ashbery pensadas desde los conceptos de obra e imagen de autor
}

IVÁN ORTEGA LÓPEZ

Universidad Nacional Autónoma de México

\section{Resumen}

En este artículo utilizo grabaciones en audio de intervenciones públicas del poeta John Ashbery para pensar la manera en la que éstas podrían relacionarse con los conceptos de obra e imagen de autor. También reflexiono sobre las posibles maneras en las que el contenido que se encuentra en internet podría modificar la manera en la que se conciben los conceptos mencionados.

Palabras clave: John Ashbery, autoría, internet, grabaciones, imagen de autor

\begin{abstract}
In this article I use audio recordings of public apparitions of the poet John Ashbery and think the ways in which these files could be analyzed using the concepts of literary work and authorial image. In this text I also dissert about the possible ways in which internet content may modify the way in which these concepts are conceived.
\end{abstract}

Keywords: John Ashbery, authorship, internet, audio recordings, authorial image 
J ohn Ashbery es un poeta comúnmente asociado con el surrealismo y el posmodernismo, así como con el grupo de poetas conocido como The New York School. El estilo de Ashbery se caracteriza por la combinación de referencias tanto a la "alta cultura" como a la cultura popular ("Daffy Duck in Hollywood"), el uso de juegos formales y de lenguaje ("Paradoxes and Oxymorons"), la explotación humorística de la rima forzada y el cliché ("Variations, Calypso and Fugue on a Theme of Ella Wheeler Wilcox"), el uso de imágenes cotidianas ("Grand Gallop"), el uso de la ironía y de frases espontáneas, reflexiones sobre la escritura ("Introduction”), frases que pueden imitar tanto la lengua vernácula como lenguajes especializados, como el de la crítica de arte, por ejemplo ("Self-Portrait in a Convex Mirror").

Nieves Alberola Crespo (2002: 71) divide la obra de Ashbery en tres etapas: Una primera en la que él mismo declara estar influido por la poesía de Stevens y Marianne Moore, de la cual deriva su primer volumen de poesía, Some Trees; en un segundo periodo quiere romper con esas influencias y decide hacer experimentos con el lenguaje, como en The Tennis Court Oath; y la tercera etapa se inicia con la publicación de Three Poems, en la que vuelve a centrarse en los procesos de la mente o en la mente como proceso. A partir de este momento su poesía entra en una fase de madurez en la que siguen estando presentes los juegos de la mente, las imágenes surreales y el lenguaje-acción. (71)

Sus primeros libros, sobre todo The Tennis Court Oath, son extremadamente experimentales, lo cual hizo que durante esos años fuera ignorado por gran parte del público y atacado por los críticos. Sin embargo, tras la publicación de Self-Portrait in a Convex Mirror en 1975, libro que ganó diversos premios (el Pulitzer, el National Book Award y el National Book Critics Circle Award), la obra de Ashbery se popularizó y en la actualidad es probablemente el poeta norteamericano vivo más conocido e influyente de Estados Unidos.

Ashbery es actualmente lo que podría considerarse un autor consagrado, de hecho, fue elegido como poeta laureado del estado de Nueva York en la década pasada. Sin embargo, la popularización de la obra de Ashbery no significa que haya dejado de ser un autor difícil. De hecho, calificativos como "hermética" o "incomprensible" siguen siendo comunes entre algunos críticos al hablar de su obra. Ashbery es una figura contradictoria, provocadora y controversial y por 
ello sumamente interesante. En este texto, me propongo hablar de la imagen de autor de John Ashbery, o al menos de la imagen que se genera de él al analizar algunas grabaciones de sus lecturas en público, siguiendo algunas de las ideas propuestas por Dominique Maingueneau. También quiero hablar sobre la información que Ashbery revela en estas intervenciones y que no aparece en ningún otro lugar de su corpus, pues considero que esta información puede arrojar algo de luz sobre su obra poética. Más que hacer un trazado general de la postura y la imagen de autor de Ashbery, me interesa mostrar cómo éstas se manifiestan al analizar ejemplos específicos. Finalmente, hablo sobre el lugar que estas grabaciones ocuparían en el corpus ashberiano, así como de la manera en la que el contenido que aparece en internet puede modificar la manera en la que se estudian la obra y la imagen de diversos autores.

Antes de continuar, me parece importante señalar cómo llegan a nosotros los comentarios que discuto en este texto. Éstos fueron hechos por el autor durante diversas lecturas en público de su obra, las cuales han sido preservadas en archivos de audio y actualmente pueden consultarse en la sección de archivos sonoros de la página, en el apartado dedicado a John Ashbery. La voz del autor, los comentarios espontáneos y no editados son aspectos importantes que se encuentran en estas grabaciones. El acervo de esta página contiene grabaciones de lecturas hechas en las décadas de 1960, 1970 y 1980, principalmente; es decir, en las décadas en las que Ashbery publica los libros de poesía que los críticos han considerado como sus obras más importantes: Rivers and Mountains (1966), The Double Dream of Spring (1970), Three Poems (1972), Self Portrait in a Convex Mirror (1975), A Wave (1984). También existen espacios similares dedicados a poetas como Joe Brainard o Robert Creeley. Por otro lado, me parece importante señalar que la aparición de un acervo de archivos sonoros de las lecturas de John Ashbery en una página como UbuWeb es significativo ya que no sólo se trata del rescate de archivos de difícil acceso sino también de que los editores del sitio alinean a Ashbery con el resto del contenido de la página: arte sonoro, música experimental, arte conceptual, revistas de difusión de grupos vanguardistas, arte callejero, textos situacionistas, archivos sobre movimientos "underground", poesía concreta, etcétera. 
Estas grabaciones juegan un papel importante a la hora de hablar de una "imagen de autor" de John Ashbery pues en ellas se pueden apreciar con detalle algunos de los aspectos que se toman en consideración para formularla: datos biográficos o seudoautobiográficos, mención de intereses estéticos, modos de comportamiento, manera de hablar (y para este aspecto la existencia de audios es de suma importancia), mención de lecturas de juventud y de autores que han influido en sus textos, así como un posicionamiento frente a su propia obra. Percatarse también del gran número de lecturas públicas que ha dado Ashbery contribuye a la formación de una imagen de autor. La gran cantidad de grabaciones tanto en video como en audio nos muestra a un autor reconocido, solicitado y que no tiene reparos en aparecer continuamente en público: es decir, no sólo es prolífico en su producción escrita sino también en apariciones públicas y en lecturas de su obra. ${ }^{1}$

Dominique Maingueneau nos dice en "Autor e imagen de autor en el análisis del discurso" que la "imagen de autor" "constituye una realidad inestable, pues es el producto de una interacción entre interventores heterogéneos" (s. a.: 51): la imagen de autor es generada tanto por el autor como por su público y la manera en la que éste se aproxima a los registros que deja el autor en diversos medios: libros, entrevistas, artículos académicos o periodísticos sobre su obra o, en nuestro caso, grabaciones de sus lecturas. Maingueneau añade que "el 'autoritas' que ha sido reconocido [es] el único suceptible de tener una 'imagen de autor"' (s. a.: 57). Es decir, para empezar a hablar de imagen de autor primero se necesita hablar de un autor que ya ha despertado interés en el público, ya sea que esté en ascenso o que ya se haya consagrado. Maingueneau

1 Geoff Ward hace referencia a lo prolífico que es Ashbery de la siguiente manera: "Recently I examined a PhD by a candidate writing on John Ashbery. The candidate noted in his preface with an absolutely heroic evenness of tone that Ashbery had published more new poetry in the four years it took to write the thesis than Philip Larkin had published during his entire lifetime" (1999: 90). La producción de obra poética de Ashbery es, para los estándares de sus contemporáneos, quizá excesiva. A ésta se suman las diversas obras teatrales, ensayos sobre literatura y arte, una novela y traducciones (quizá la más importante sea su versión de las Iluminaciones de Rimbaud). La enorme cantidad y la gran variedad de la obra de Ashbery permite que ésta pueda ser abordada desde múltiples ámbitos. 
continúa: "Ciertos autores acceden al estatuto de 'autoritas' mayor o de 'gran autor'[...] Su figura es tan prominente que incluso se publican textos que ellos no habían destinado a la publicación: borradores, correspondencia privada, tareas escolares, diarios, cuadernos, etc. [...] en el caso de los 'grandes autores' decisiones editoriales, basadas en operaciones de interpretación, pueden hacer que un género que no estaba destinado a la publicación contribuya a forjar la imagen de autor" (s. a.: 58). La formación y el interés por la imagen de un autor viene siempre después del éxito de su obra (ya sea éste en vida o póstumo), y una vez que un autor es consagrado todo tipo de textos suyos despiertan interés.

Por otra parte, el "gran autor" es susceptible de que se le dé por sentado; es decir, que textos nuevos suyos se publiquen y aplaudan, independientemente de su calidad, sin que necesariamente sean leídos. Geoff Ward comenta al respecto de Ashbery:

He must wonder if people now read, or merely dip into, or now feel that they have already read, each successive book. [. . . 'Like a sentence' appears in And the Stars were Shining, only to turn up again word-for-word in Can You Hear, Bird. How many people noticed? Does it really matter? Is Ashbery's canonization now so achieved a phenomenon that were he to publish chunk of the telephone directory as his next book [...] it would not alter one line in a profile that is now set in stone? (1999: 91)

La obra de Ashbery corre el riesgo de que sea dada por sentada no sólo debido a la consagración del autor sino al amplio volumen de ésta. La obra periférica de Ashbery es también de tamaño considerable. Sin embargo, a pesar de que podría pensarse como innecesaria o de poca importancia, pues muchas veces se presenta en fragmentos periféricos o secundarios, en las grabaciones en audio podemos encontrar información importante para entender no sólo a Ashbery como autor o personaje, sino también poemas suyos que podrían considerarse oscuros o menores.

Ciertamente, el acervo de archivos en audio de UbuWeb no despertaría interés de no ser John Ashbery un poeta consagrado. Aunque al publicarlos en realidad se asumen pocos riesgos editoriales, estos archivos son indicadores de la existencia de un interés por la obra de Ashbery que va más allá de los textos 
impresos. Sin embargo, si bien resultan fuentes de información importante acerca de la obra de Ashbery, ocupan un lugar más bien periférico en el corpus de este autor. Lo que me interesa rescatar de estas grabaciones son las declaraciones que hace, más que la lectura de los poemas mismos, pues a pesar de que se trata en muchos casos de simples añadidos a los poemas, observaciones espontáneas e incluso anécdotas superficiales, considero que las declaraciones hechas en estas lecturas tienen valor por sí mismas.

Estas declaraciones en muchos casos suelen proporcionar información sobre poemas específicos, así como también suelen mostrar algunas actitudes de Ashbery frente a su obra y frente al público. Julián Jiménez Hefferman, en el prólogo a su traducción de Tres poemas, señala la importancia que tuvieron las declaraciones de Ashbery, tanto en entrevistas como lecturas, para los críticos de su obra: "Pese a la perplejidad crítica, Ashbery nunca ocultó los secretos de su receta. Eso sí, los fue dosificando en entrevistas y declaraciones dispersas. Si reunimos todo ese material [...] obtenemos un conjunto de pistas útiles, que no explican los poemas, pero al menos los replican, los reverberan" (Jiménez Hefferman, 2004:10). Hefferman habla de declaraciones hechas en entrevistas publicadas en revistas de difusión limitada, en conversaciones o en las lecturas de su obra, las cuales resultaron clave para aproximarse a los poemas y para entender los procesos de escritura del poeta. No es sino hasta la publicación, propiciada por internet, de materiales como los que discuto en este texto, que ciertos comentarios de Ashbery pudieron alcanzar un público más amplio. También considero que parte de la importancia de estas declaraciones recae en el parecido temático y estilístico que guardan con la obra poética de Ashbery. Al igual que los poemas de este autor, las declaraciones están compuestas por una combinación de lenguaje coloquial, comentarios eruditos, anécdotas absurdas y observaciones herméticas, así como de pasajes que reflexionan sobre la escritura poética.

La primera frase del título de este texto proviene de un comentario hecho por John Ashbery durante una lectura en público de su obra poética en el Center of Contemporary Arts of Santa Fe en noviembre de 1985. Este comentario fue hecho después de concluir la lectura de su poema "Worsening Situation" cuyos últimos versos dicen "My wife / Thinks that I'm in Oslo-Oslo, France, that is" 
(Ashbery, 2008: 429). Lo que Ashbery dice al concluir su poema es lo siguiente: "Sometimes I've been asked after reading that poem 'Is there an Oslo, France, mr. Ashbery?', and I have always said: I don't think so but I don't have a wife either. Both of these are examples of 'poetic licence' which is much easier to accept as a concept than when you are actually confronted with an example of it". En este comentario puede apreciarse el tono irónico con el que Ashbery suele referirse a su labor como poeta; también se le percibe un tanto burlón respecto a la incomprensión de su obra. Otro ejemplo de actitudes similares puede verse en un comentario hecho antes de comenzar la lectura de su poema "To a Waterfowl" en una lectura realizada en 1966: "Excuse me if I smoke while you can't. There has to be some advantage of being a poet. [...] This poem is made out of lines from another poems, some of which you may recognize, and I discovered after I wrote it that this is a form which was used in Italy during the reinassance, It's called a cento". Al mismo tiempo que hace chistes acerca de la posición del poeta en la sociedad, explica ciertos aspectos del proceso de elaboración de su poema, el cual consiste en líneas tomadas de poemas de otros autores, como Wordsworth, Yeats y Eliot.

Estos comentarios son dos ejemplos de los muchos que John Ashbery suele hacer durante la lectura de su obra en público. En ellos pueden apreciarse algunas concepciones que tiene Ashbery sobre su obra en particular y sobre la escritura de poesía en general, así como de la recepción de ésta. Junto a anécdotas de distintos tipos, Ashbery suele mencionar las circunstancias en las que escribió algunos de sus poemas, proporcionando nuevas perspectivas para abordarlos. Estas declaraciones difieren de las hechas en entrevistas ${ }^{2}$ en diversos aspectos, aunque quizá el más importante sea que se refieren a poemas en específico, y en muchos casos se trata de poemas "menores" o poco conocidos ("He", "The IceCream Wars", "Glazunoviana”, entre otros) en lugar de limitarse a hablar de su

2 Las entrevistas a Ashbery también abundan. Si bien para este trabajo las he dejado de lado, de entre ellas me gustaría señalar la concedida a The Paris Review como una de las más significativas, así como la entrevista concedida a The Library of America, que acompañó la edición de Collected Poems 1956-1987. Ambas entrevistas pueden encontrarse en línea. 
obra en rasgos generales o de sus poemas más conocidos (como "Self-Portrait in a Convex Mirror”, “Clepsydra”, “The System”).

Estas declaraciones establecen una relación activa con la obra y afectan la manera en la que uno podría percibir a John Ashbery como autor. Tomemos como ejemplo lo que dice John Ashbery antes de leer un poema en mayo de 1976:

This is one [poem] that I can remember writing. It's called "Daffy Duck in Hollywood". [...] I went to a program of animated cartoons at a museum in New York a year or so ago and at the same time I was reading Paradise Lost and there was a Daffy Duck cartoon in which you see the pencil of the cartoonist sort of adding extra limbs and erasing his head and various parts as he keeps complaining about this and I somehow subconsciously associated this with the idea of God in the first book of Paradise Lost, who has always seemed to me rather comically conspicuous by his absence while all the debate is going on down there and, in fact, I put there a couple of lines from Paradise Lost. So I seem somehow to associate Satan with Daffy Duck although I had no idea that I was doing this at the time, they are somehow alike [...] It's got a lot of characters from books and movies and a lot of French words. I don't believe in really explaining very much but the French carte du tendre is an allegorical map of the country of love [...].

Durante unos momentos más, Ashbery continúa explicando el significado de algunas de las frases en francés que aparecen a lo largo del poema. Más que de una explicación del poema, se trata de una lista de ingredientes que éste contiene, así como de una breve mención de las circunstancias en las que fue escrito. Por la diversidad de contenidos, este pasaje es muy similar a las prosas "explicativas" que Gerardo Deniz, poeta que en momentos es igual o más hermético que Ashbery, elaboró para acompañar algunos de sus poemas, prosas que en realidad son listas que explican las diversas referencias que hace en sus textos, sin explicar los poemas en sí. ${ }^{3}$ En esta cita puede apreciarse cómo Ashbery

3 Tales notas están contenidas en Visitas guiadas así como en el volumen antológico Mansalva. La descripción que hace Ashbery de la concepción y elaboración de sus textos trae también 
no tiene reparos en mezclar la "alta cultura" con la cultura de masas al hablar de sus influencias, en lo cual puede verse la postura que toma Ashbery como autor respecto a los diversos focos de la cultura. Esta postura podría incluso considerarse provocadora, sobre todo si tomamos en cuenta que la lectura se lleva a cabo en un auditorio de la Universidad de Harvard.

El tono con el que habla es casual; sin embargo, en lo que dice pueden apreciarse diversos cambios de temas y de registros, del anecdótico al erudito. Estas derivas también son comunes en poemas suyos, como "Self-Portrait in a Convex Mirror" o los poemas en prosa de Three Poems. Ashbery describe pero no pretende forzar ninguna interpretación o posible lectura de su poema (gesto que también puede analizarse como un ejemplo de postura de autor), lo cual puede estar relacionado con la idea barthiana de la "muerte del autor", es decir, del fin del autor como dador del sentido último de sus textos. Sin embargo, la presencia del autor mismo en estas declaraciones y lecturas hace que esto resulte paradójico. Al oír estas declaraciones antes de escuchar (o leer) el poema, el espectador (o el lector) se acerca a él con más herramientas, sin por ello tener una lectura guiada por el autor. Esta introducción ilustra lo que Nieves Alberola Crespo dice respecto de algunos poemas de Ashbery: son "poemas que buscan ser experiencias y no aportar un significado concreto alguno" (2002: 161).

En estas declaraciones, Ashbery muestra, más que el camino a seguir para leer sus poemas, el camino que él siguió para componerlos, lo cual puede resultar de ayuda en algunos casos para acercarse al poema. Lo que también hace ver es la variedad de métodos con los que trabaja. Si hacemos caso a lo que el poeta dice, vemos que parece que para cada poema utilizó un proceso de escritura distinto. Por ejemplo, sobre el poema "He", de su primer volumen de poemas Some Trees, dice: "It's a poem that I got the idea for from a dream. I dreamt that I have written a poem called 'He' in which all of the lines began with the pronoun 'he'. And then I woke up and I wrote the poem". De esta explicación, breve y simple, se pueden desprender varias conclusiones. La primera es que

a la mente "Cómo escribí algunos de mis libros", de Raymond Roussel, una de las principales influencias de Ashbery. 
pareciera que Ashbery se está burlando de la anécdota sobre la composición del poema "Kubla Khan" de Samuel Taylor Coleridge; no sería la primera vez que Ashbery alude o parodia a algún poeta romántico inglés. ${ }^{4}$ Otra conclusión es que apunta a una asociación con la estética surrealista y con algunos escritores de esta escuela, con la que se ha asociado a Ashbery. También hay que considerar la manera en la que Ashbery habla constantemente sobre el poco tiempo que dedica a la escritura de sus poemas y al poco trabajo que le cuesta elaborarlos. Hablar sobre métodos compositivos o de la escritura misma es también tema de algunos de sus poemas, como puede verse en la segunda estrofa de su poema "Ode to Bill": "What is writing? / Well, in my case, it's getting down on paper / Not thoughts, exactly, but ideas, maybe; / Ideas about thoughts..." (Ashbery, 2008: 461). El tono de este pasaje, que imita algunos giros del habla cotidiana, hace ver que incluso estilísticamente las declaraciones y los poemas tienen una fuerte relación.

Otro ejemplo de un poema que según el propio Ashbery elaboró en poco tiempo puede hallarse en "Pyrography". Ashbery dice, respecto a este texto:

This one [...] was actually commissioned by the Department of the Interior for a catalogue of American Landscapes which is currently in Washington and is going to circulate around the country for its bicentennial exhibition. [...] There were two poems in the catalogue and this was supposed to be supplied by somebody else who sort of hitched for a year, and I was asked to do it in about a day, and I said I didn't write that way and that the bicentennial didn't mean anything in particular to me. Then he told me how much he was paying and I radically revised my aesthetic program.

4 La relación entre Ashbery y el romanticismo inglés es abordada en dos de los once ensayos contenidos en Romanticism and Postmodernism: "Teleotropic Syntax in Ashbery and Wordsworth" de Geoff Ward, y "Uprooting the Rancid Stalk: Transformations of Romanticism in Ashbery and Ash" de Stephen Clark, quien incluso recoge esta declaración de Ashbery: "All my stuff is romantic poetry rather tan metaphysical or surrealist" (Ashbery citado en Clark, 1999: 157). 
La referencia a la escritura de poemas en cortos plazos es común tanto en estas declaraciones como en diversas entrevistas. Esta facilidad para la escritura (el mismo Ashbery asegura que pocas veces corrige) quizá sea la explicación del gran número de volúmenes que abarca su obra poética. Ashbery recalca este aspecto hasta extremos casi arrogantes. No obstante, como en muchos otros aspectos sobre la obra de Ashbery, habrá que preguntarnos si no estará solamente actuando o interpretando un papel. Esto entra en relación con lo que Maingueneau llama "las tres dimensiones de la noción de autor", la segunda de las cuales es presentada de la siguiente manera: "La segunda dimensión es la del 'autor-actor.'Ésta corresponde a aquel que, al organizar su existencia en torno a la producción de textos, debe administrar una trayectoria, una carrera. No se trata únicamente de una profesión, pero sí de una actividad, de un comportamiento" (Maingueneau, s. a.). Al escuchar a Ashbery hablando sobre sus poemas, uno termina por preguntarse hasta qué punto está actuando, asumiendo su papel de "poeta" o de "autor consagrado" o incluso de "virtuoso", "máquina escribiente" o "superdotado".

Cabe también pensar si no estará solamente burlándose de todo esto, o preguntarse qué tan ciertas son las anécdotas que cuenta. Su actitud resulta ambigua y es imposible optar por alguna conclusión. Maria Stoopen comenta, en su nota introductora al texto “¿Qué es un autor?” de Michel Foucault: “En los discursos provistos de un autor, los signos gramaticales que remiten al autor de un texto no aluden a su persona, sino a un alter ego, distante y distinto de él. Así, los discursos provistos de esta function implican una pluralidad de ego y tal función da lugar a varias 'posiciones sujeto"' (Stoopen, 2009: 109). Si bien Stoopen se refiere sobre todo a discursos escritos, como los poemas de Ashbery, en los cuales de hecho es posible encontrar una gran cantidad de alter egos, también puede pensarse en relación con las declaraciones de Ashbery, pues al hacerlas pareciera también estar interpretando diversos papeles, usando diferentes máscaras. Con la postura que toma frente a su producción, así como frente al público, cabe decir lo que Keith Cohen dice respecto al tono en los poemas de Ashbery: "The voice of the poems seems at one moment to be mouthing the discourse, at the next time to be mocking it [...] it is like trying to differentiate between a well-molded graduate from Harvard Biseness School and a come- 
dian's impression of a business-man" (Cohen citado en Clark, 1999: 159). Sobre el podio, fumando mientras que el público no puede, John Ashbery, interpreta diversos papeles: el del poeta John Ashbery, el del poeta laureado del estado de Nueva York, el del poeta antisolemne, el del defensor de la "otra tradición", el poeta de la lengua vernácula estadounidense, el del comediante que estudió negocios en Harvard, sin que podamos distinguir realmente la diferencia entre cada uno de estos papeles.

En esa intervención al respecto de "Pyrography", podemos de nuevo apreciar a un Ashbery que ironiza, con humor, sobre su trabajo como poeta. El humor forma parte importante de la obra de Ashbery, y considero que tanto su presencia como su ausencia, en la obra y en las apariciones públicas, son constitutivas de la elaboración de una "imagen de autor". Como se vio anteriormente, el humor está presente también en algunas de las declaraciones que hace Ashbery en sus lecturas en público. Contar con archivos en audio resulta importante para hablar de humor en estas circunstancias ya que en éstos puede apreciarse la respuesta del público a los momentos humorísticos de los poemas de Ashbery; por ejemplo, las tres líneas rimadas del poema "Variations, Calypso and Fugue on a Theme of Ella Wheeler Willcox": "But of all the sights that were seen by me / In the east or West, on land or sea, / The Best was the place that is spelled H-O-M-E” (Ashbery, 2008: 191), o bien este pasaje del collage de versos que hace en "To a Waterfowl": "Go, lovely rose, / This is no country for old men. The young / Midwinter spring is its own season" (Ashbery, 2008: 923). Ashbery adopta en ocasiones el mismo tono juguetón y humorístico de sus poemas, y a momentos cabe preguntarse si lo que busca no es un efecto cómico a toda costa. Un ejemplo claro de esto es la introducción que hace a su poema "The Ice-Cream Wars":

Concerning this poem somebody asked me recently what the title meant or what I meant by the title, and I said: I hadn't meant anything by the title. However, a few months ago an ice-cream truck blew up in lower Manhat$\tan$ and injured a lot of people but nobody...people still don't know why... what caused the explosion and at the time it was mentioned in a newspaper that this may be a revival of the "ice-cream wars" of the early sixties when rival ice-cream distributors were going around and blowing up each 
other's ice-creamtrucks, which I never heard of but I claim that it's part of the collective unconscious.

Esta intervención consiste en sólo una anécdota, con humor negro, que, a diferencia de las anteriores aquí citadas, no aporta nada de información respecto al poema que precede. Sin embargo, pensada desde la idea de imagen de autor, nos aporta algo de información: que Ashbery procura construir una imagen autoral alejada de la solemnidad con la que podría asociarse a la poesía o a un hombre de letras en general. En esta intervención también se muestra como un autor que, como ya se comentó, no pretende hablar de posibles interpretaciones de sus poemas ni imponerlas. Por otra parte, estilística y temáticamente este fragmento es muy similar a algunos poemas ashberianos e incluso podría figurar sin problemas en alguno. Comparemos tanto el tono como el contenido de esta anécdota con algunas líneas de su poema "These Lacustrine Cities": "Much of your time has been occupied by creative games / Until now, but we have all-inclusive plans for you. / We had thought, for instance, of sending you to the middle of the desert" (Ashbery, 2008: 125).

Como ya mencioné, considero que parte de la importancia que podrían tener estas declaraciones se debe no sólo a la información que aportan respecto a la obra, la postura o la imagen de autor de Ashbery, sino también al gran parecido que guardan con su obra poética. Dominique Maingueneau habla de la importancia de buscar la coherencia entre autor "real" y obra al hablar de imagen de autor. Por su parte, Nieves Alberola Crespo señala que uno de los principales intereses estéticos de Ashbery en algunos de sus poemas es mostrar qué tan poético puede llegar a ser el lenguaje más prosaico. Julián Jiménez Hefferman, por su parte, habla sobre la inclusividad que caracteriza a la obra del poeta. Otros rasgos que menciona son la reflexión, la espontaneidad y el coloquialismo (Hefferman, 2006: 14), los cuales también pueden apreciarse en sus aclaraciones. El mismo Ashbery en una entrevista declaró: "muchos de mis poemas tienen su origen en lo que oigo decir por la calle en Nueva York, en la lengua vernácula" (Ashbery, 2006: 10). Quizá si se tratara de otro autor, con diferentes intereses estéticos, no podría establecerse una conexión como la que hay entre los poemas de Ashbery y las declaraciones con las que los acompaña 
en sus lecturas en público. Conceptos como imagen o postura de autor no sólo permiten utilizar este tipo de material sino que también hacen necesario que se tengan en consideración al hablar de un autor. Un acercamiento "textualista" a la obra de Ashbery dejaría de lado este tipo de materiales, a pesar de que éstos proporcionan información valiosa para acercarse a la obra del poeta.

Como mencioné anteriormente, me parece pertinente abordar la cuestión del lugar que ocuparían estas grabaciones, sobre todo las declaraciones que en éstas hace Ashbery, en el corpus del poeta, así como la manera en la que abren nuevas posibilidades para pensar el concepto de obra. Este lugar es evidentemente periférico, pero estas declaraciones guardan con la obra central de Ashbery una relación muy estrecha. Sin embargo, el ser archivos sonoros y no textos escritos vuelve problemática su aceptación como componente de la obra de este poeta. Por otro lado, como ya señalé, estas declaraciones guardan un parecido estilístico y temático con los poemas de Ashbery, lo cual podría acreditarlas como obra. ${ }^{5}$

Michel Foucault, en su texto “¿Qué es un autor”, se pregunta: “QQué es, pues, esa curiosa unidad que se designa con el nombre de obra? ¿De qué elementos está compuesta? Una obra, ¿no es aquello que escribió aquel que es un autor? Se ven surgir dificultades". Una definición estrecha como ésta ciertamente dejaría de lado los archivos analizados en este texto, a pesar de que, como he mostrado, no sólo guardan un parecido estilístico con los poemas de Ashbery, sino que también proporcionan información importante. Foucault continúa:

5 En su prólogo a la antología de entrevistas de Andy Warhol, Reva Wolf trae a colación una serie de cuestionamientos acerca de la entrevista, que me parecen análogos a los que hago acerca de las grabaciones de las lecturas de Ashbery: "Pese a que otros artistas se han valido de la entrevista para crear arte [...], ningún artista se ha aproximado al grado de energía que dedicaba Warhol a esta actividad y ninguno ha sido capaz de extraerle tanto jugo. Warhol fue capaz de dar nuevos, y a menudo múltiples, sentidos a las palabras más prosaicas [...] ¿Acaso son arte estas entrevistas? Vistas en el contexto del conjunto de la obra de Warhol en todos los medios, diría que la respuesta debe ser que sî" (Reva, 2010: 32). La completa coherencia de las entrevistas que concedía Warhol con el resto de su obra equivale, me parece, a la coherencia entre las declaraciones de Ashbery y sus poemas. 
Cuando se emprende la publicación de las obras de Nietzche, por ejemplo, ¿en dónde hay que detenerse? Hay que publicar todo, ciertamente, pero ¿qué quiere decir ese "todo"? Todo lo que Nietzche publicó, de acuerdo. ¿Los borradores de sus obras? Ciertamente. ¿Los proyectos de aforismos? Sí [...] Pero cuando en el interior de un cuaderno de aforismos se encuentra una referencia, la indicación de una cita o de una dirección, una cuenta de lavandería ¿Obra o no obra? ¿Y por qué no? Y esto indefinidamente (2009: 117).

Ciertamente las grabaciones prueban que fue Ashbery el emisor de aquellas declaraciones, pero el que sean grabaciones y no textos escritos los hacen documentos problemáticos. La cuestión de la publicación ha quedado superada: los archivos ya están a disposición del público. Sin embargo, quedan otras cuestiones por resolverse: ¿hasta dónde se puede extender la autoría de Ashbery en particular o la autoría de cualquier otro escritor en general? ¿Todas las declaraciones las hace como "autor" o como "persona"? ¿Cómo es posible distinguir dónde acaba uno y comienza la otra?

El modo de publicación de estas grabaciones, en una página de internet, trae a la mente lo que Maingueneau menciona al final de su artículo: "Hoy en día nos encontramos en una fase de transición entre el régimen tradicional de dominación del impreso y un régimen 'digital' en perpetua transformación. Resulta bastante difícil determinar si internet va a crear o no... nuevas formas de singularización de los textos que coexistan con los precedentes" (s. a.: 65). Internet facilita la publicación de material que de otro modo sería impublicable, como es el caso de los archivos con los que trabajé para elaborar este texto. ${ }^{6} \mathrm{La}$ disponibilidad de este tipo de materiales hace que éstos puedan ser tomados en consideración al hablar tanto del autor como de su personaje público, lo cual proporciona datos que no podrían encontrarse en ninguna otra parte de la obra. Adriana de Teresa Ochoa comenta: "es posible constatar la profunda transformación que ha significado el desarrollo de nuevas tecnologías, las

6 UbuWeb cuenta también con una colección de libros en PDF que lleva el nombre de Publishing the Unpublishable, casi todos pertenecientes al género de la poesía concreta. 
cuales han creado formas inéditas de producción, circulación y apropiación de lo escrito" (2010: 124), a lo que también tendríamos que añadir otro tipo de documentos, no necesariamente escritos, pero que tienen una estrecha relación con la literatura.

La difusión que tienen hoy en día archivos como los que se discuten en este trabajo hace pensar en nuevos modos de concebir la autoría y la publicación. El autor ya no es un ser tan distante cuyo único rostro o voz se encuentra en lo que escribe o lo que se escribe sobre él. Archivos tanto de video como de audio de presentaciones públicas o entrevistas a escritores proliferan en la red, así como versiones digitales de sus obras. Independientemente de si el autor está consciente de ello o no, sus declaraciones o sus comportamientos quedan registrados y son difundidos. Conceptos como imagen de autor hacen que este tipo de registros puedan ser estudiados. Roger Chartier dice: "Disociados de los soportes en los que tenemos la costumbre de encontrarnos (el libro, el periódico) los textos estarían de ahora en adelante consagrados a una existencia electrónica" (2010: 17).

Estamos en un momento de transición en el que conviven diversos modos de autoría. No se trata sólo de autores nuevos que están utilizando internet para difundir su obra, sino también de las facilidades que proporciona internet para que aparezcan archivos relacionados con autores que anteriormente resultaban inaccesibles para el público. ${ }^{7}$ Chartier aboga por un sistema en el que los diversos modos de autoría y difusión convivan sin contraponerse o sin intentar sustituir el uno al otro. "La biblioteca del futuro - dice- debe ser también el lugar en que se pueda mantener el conocimiento y la comprensión de la cultura escrita en las formas que han sido y son todavía mayoritariamente las suyas hoy en día" (2010: 30).

Al pensar en conceptos como autor, obra y autoría es necesario tener en cuenta cada vez una mayor cantidad de aspectos. En este sentido, Ashbery me

7 Un ejemplo interesante es la publicación de los facsímiles de los cuadernos manuscritos del poeta peruano Luis Hernández, en los que se resalta la importancia de las versiones manuscritas de su obra pues va acompañada de dibujos. De igual manera el poeta utiliza distintos colores para cada estrofa, entre otras cosas. 
parece un buen ejemplo de la manera en que conviven estos dos sistemas: por un lado, la obra impresa (los libros de poesía, las entrevistas, los ensayos) y, por otro, los diversos videos de lecturas, de entrevistas o las grabaciones en audio de éstas.

\section{Bibliografía}

Alberola Crespo, Nieves (2002): La escuela de Nueva York, John Ashbery y la nueva poética americana. Barcelona: Universidad Jaume I.

Ashbery, John (2004): Tres Poemas (trad. y pról. Julián Jiménez Hefferman). Barcelona: DVD.

Ashbery, John (2006): Autorretrato en espejo convexo (trad. y pról. Julián Jiménez Hefferman). Barcelona: DVD.

Ashbery, John (2008): Collected Poems, 1956-1987 (ed. Mark Ford). Nueva York: The Library of America.

Ashbery, John [s. f.]: "Daffy Duck in Hollywood", "He”, "Pyrography", “The Ice-Cream Wars", “To a Waterfoul”, “Worsening Situation”. En UbuWeb.

Chartier, Roger (2010): "Del códice a la pantalla: trayectorias de lo escrito". En Adriana de Teresa Ochoa (coord.), Circulaciones: trayectorias del texto literario. México: Bonilla Artigas / Universidad Nacional Autónoma de México, pp. 17-30.

Clark, Stephen (1999): “Uprooting the Rancid Stalk': Transformations of Romanticism in Ashbery and Ash”. En Edward Larrissy (ed.), Romanticism and Postmodernism. [Cambridge] Cambridge University Press, pp. 157-178.

Foucault, Michel (2009): “¿Qué es un autor?”. En María Stoopen Galán (coord.), Sujeto y relato. Antología de textos teóricos. México: Universidad Nacional Autónoma de México, pp. 109-132.

Jiménez Hefferman, Julián (2004), “Prólogo”. En John Ashbery, Tres poemas (trad. y pról. Julián Jiménez Hefferman). Barcelona: DVD. 
JimÉnez HefFerman, Julián (2006): “Prólogo”. En John Ashbery, Autorretrato en espejo convexo (trad. y pról. Julián Jiménez Hefferman). Barcelona: DVD.

Maingueneau, Dominique [2014]: "Autor e imagen de autor en el análisis del discurso". En Juan Zapata (comp.) La invención del autor. Medellín: Universidad de Antioquia, pp. 49-66.

Stoopen Galán, María (2009), [Nota introductoria a Michel Foucault]. En María Stoopen Galán (coord.), Sujeto y relato. Antología de textos teóricos. México: Universidad Nacional Autónoma de México, p. 109.

Teresa Ochoa, Adriana de (2010): "La función del autor en la circulación literaria”. En Adriana de Teresa Ochoa (comp.), Circulaciones: trayectorias del texto literario. México: Bonilla Artigas / Universidad Nacional Autónoma de México, pp. 105-127.

WARD, Geoff (1999): "A Being All Alike? Teleotropic Syntax in Ashbery and Wordsworth". En Edward Larrissy (ed.), Romanticism and Postmodernism. [Cambridge] Cambridge University Press, pp. 86-97.

WARHol, Andy (2010): Entrevistas (introd. Reva Wolf, ed. Kenneth Goldsmith). Barcelona: Blackie Books.

Wolf, Reva (2010): “Introducción”. En Andy Warhol, Entrevistas (introd. Reva Wolf, ed. Kenneth Goldsmith). Barcelona: Blackie Books. 